\title{
Técnicas de manejo y conservación de suelos en escenarios cafetaleros en zonas susceptibles a erosión
}

\section{Techniques of soil management and conservation in coffee scenarios in areas susceptible to erosion}

\author{
Richard Palma Ponce ${ }^{1 *}$, Jesús De los Santos Pinargote Choez ${ }^{1}$ y Lucy Maritza García Lucas ${ }^{1}$ \\ ${ }^{1}$ Universidad Estatal del Sur de Manabí \\ *richard.palma@unesum.edu.ec
}

DOI: https://doi.org/10.26871/killkana_tecnica.v3i1.470

\begin{abstract}
Resumen
El cantón Jipijapa se encuentra atravesado por un sistema montañoso que forma parte de la cordillera costanera del litoral ecuatoriano, motivo por el cual presenta topografía irregular con pendientes muy prolongadas. Esto conlleva a que los sistemas agroforestales predominantes en la zona (café-árboles), se desarrollen en zonas con riesgo de erosión y deslaves. El objetivo del trabajo fue identificar los perfiles que presentan los suelos y definir acciones que coadyuven a mejorar las condiciones de manejo y conservación en 17 escenarios cafetaleros, los cuales se encuentran ubicados en diferentes zonas susceptibles a erosión de las parroquias. Los perfiles de suelos se determinaron mediante la construcción de calicatas con dimensiones de 1,2 metros cúbicos, definiendo las principales características físicas en cada horizonte, complementándolos con resultados de análisis químicos de laboratorio a partir de otro proyecto de investigación efectuado por la UNESUM. Se construyeron terrazas individuales como estrategias de conservación de suelo en las plantaciones de café y se elaboró compost tipo bocashi modificado y caldo microbiológico, como alternativa de manejo, orientada a recuperar la fertilidad de los suelos en las 17 fincas vinculadas al proyecto. El 41,2\% de los suelos resultaron ser profundos $(>100 \mathrm{~cm}$ de profundidad); un $35,3 \%$ suelos moderadamente profundos $(>60<100 \mathrm{~cm}$ de profundidad) y $23,5 \%$ suelos poco y muy poco profundos. Referente a la textura de los suelos se determinó que el 76,5\% tienen tendencia franca, predominando ligeramente los francos arcillosos, con el $29,4 \%$, considerados aptos para la agricultura.
\end{abstract}

Palabras clave: perfil de suelo, textura, compost, fincas, terrazas individuales.

\begin{abstract}
The Jipijapa canton is crossed by a mountainous system that is part of the Ecuadorian coastal mountain range. For this reason, it has irregular topography with very steep slopes. Consequently, the predominant agroforestry systems in the area (coffee-trees) grow in regions at risk of erosion and landslides. This project was aimed to identify both, the soil features and the actions that contribute to the improvement of soil management as well as to its conservation conditions in 17 coffee plantations located in different areas of this parish, which are at risk of erosion. Soil features were determined through the construction of 1.2 cubic meter trial pits. The main physical characteristics on each horizon were defined and complemented with the results of laboratory chemical analysis results corresponding to another research project that was carried out by UNESUM. Individual terraces were built in the coffee plantations as a strategy for soil conservation. Modified bocashi type compost and microbiological broth were made as management alternatives aimed to recover soil fertility in the 17 farms linked to the project. $41.2 \%$ of the soils were found to be deep (>100 cm deep); $35.3 \%$ moderately deep soils $(>60<100 \mathrm{~cm}$ deep $)$ and $23.5 \%$ shallow and very shallow soils. Regarding soils texture, it was determined that $76.5 \%$ have a frank tendency, with a slight predominance of clay loams, with $29.4 \%$, which are considered suitable for agriculture.
\end{abstract}

Key words: soil features, texture, compost, farms, individual terraces.

\section{INTRODUCCIÓN}

La propuesta académica de la Universidad Estatal del Sur de Manabí "UNESUM" se fundamenta en la articulación de la docencia, la investigación y la vinculación con la sociedad, convirtiéndose en agente de desarrollo en la zona sur de Manabí, ejecutando diferentes proyectos de desarro- llo e innovación con la participación activa de la sociedad civil armonizando actividades con estudiantes y docentes universitarios. En este contexto el rediseño curricular de la carrera de Ingeniería Forestal, contempla la ejecución del proyecto de vinculación denominado "Técnicas de manejo y conservación de suelos en sector de riesgos de erosión, 
beneficios del recurso forestal maderable y no maderables aplicadas en zonas susceptibles".

Las actividades vinculadas al manejo agroforestal, intervienen directamente sobre los componentes del medio ambiente, entre ellos el suelo, poniendo en riesgo la conservación del mismo, sobre todo cuando se realiza en zonas susceptibles a la erosión, como pendientes pronunciadas.

El cantón Jipijapa El proyecto tiene como zona de influencia los sitios donde están ubicados los escenarios cafetaleros (60) que viene impulsando la UNESUM en cantón Jipijapa, Provincia de Manabí; y beneficiará en una primera etapa a 23 familias, dedicadas a la producción cafetalera.

El cantón Jipijapa está ubicado en el extremo sur occidental de la provincia de Manabí, a 403 kilómetros de Quito capital del Ecuador, tiene aproximadamente una extensión de $1.420 \mathrm{Km} 2$. Se encuentra atravesada por un sistema montañoso que forma parte de la cordillera costanera del litoral ecuatoriano, motivo por el cual presenta topografía irregular con pendientes muy prolongadas. Esto conlleva a que los sistemas agroforestales predominantes en la zona (café-arboles), se desarrollen en zonas con riesgo de erosión y deslaves.

La principal actividad de este cantón es la producción y exportación de café, además del comercio en sus diferentes formas y la artesanía. Según la clasificación climática de Köppen-Geiger, Jipijapa se encuentra en la zona climática de Bosque Semi-húmedo "BSH", con una temperatura media anual de $23.7{ }^{\circ} \mathrm{C}$ y precipitaciones promedios de $537 \mathrm{~mm} / \mathrm{año} \mathrm{[1].} \mathrm{Duicela} \mathrm{[2],} \mathrm{manifiesta} \mathrm{que} \mathrm{para} \mathrm{que} \mathrm{el}$ cafeto se desarrolle y obtenga una producción adecuada, requiere de suelos profundos y medianamente profundos, textura de tendencia francos y una nutrición equilibrada de macronutrientes ( $\mathrm{N}, \mathrm{P}, \mathrm{K}, \mathrm{S}, \mathrm{Ca}, \mathrm{y} \mathrm{Mg}$ ) y micronutrientes (Zn, $\mathrm{Cu}, \mathrm{Fe}, \mathrm{Mn}, \mathrm{Mo}, \mathrm{Cl}, \mathrm{Na}, \mathrm{B})$.

\section{ANTECEDENTES}

Jaramillo (2002), manifestó que la erosión es la disgregación de materiales sólidos del suelo por cualquier agente y mecanismo, entre ellos la lluvia el viento y actividades antrópicas, por lo que se hace necesario y urgente ser eficientes en su uso y manejo, con prácticas agrícolas sostenibles y amigables con el medio ambiente

Es necesario identificar y entender la clase de proceso de degradación (Erosión, movimiento en masa), los mecanismos de falla, las causas y efectos con el fin de planificar las actividades de prospección, la toma de muestras, la exploración del sitio del problema, analizar los perfiles del suelo, realizar el diagnóstico y plantear el tipo de solución. No entender el proceso, el mecanismo y las causas de un proceso de inestabilidad es dar un inicio incierto, que lleva a una posible solución poco práctica.

Las prácticas de conservación de suelo contribuyen a mejorar las condiciones actuales en cada sitio, sin provocar cambios que pongan en riesgo su productividad. Por lo tanto, es necesario conocer el conjunto de propiedades y condiciones que definen la productividad y el comportamiento mecánico del suelo, para tomarlas en consideración durante su aprovechamiento (Raudes y Sagastume, 2009).

El proyecto se desarrolla en los escenarios cafetaleros que viene impulsando la UNESUM ubicados en zonas de riesgo del cantón Jipijapa, vinculando a 17 familias dedicadas a la producción cafetalera, con el objetivo de conocer los perfiles de suelo y capacitar a los agricultores en técnicas de manejo y conservación de suelo, como estrategia para prevenir y contrarrestar la erosión de este importante recurso.

El objetivo del trabajo fue identificar los perfiles que presentan los suelos y definir acciones que coadyuven a mejorar las condiciones de manejo y conservación en 17 escenarios cafetaleros, ubicados en diferentes zonas susceptibles a erosión de las parroquias.

\section{MATERiales Y MÉtodos}

El proyecto se desarrolló en 17 escenarios cafetaleros ubicados en zonas de riesgo de erosión de la parroquia El Anegado y en la parte rural de la parroquia San Lorenzo de Jipijapa, en la Provincia de Manabí.

La ejecución del proyecto consistió en el trabajo en equipo entre docentes, estudiantes, técnicos del programa de vinculación con la sociedad de la UNESUM y agricultores. Se realizaron reuniones de trabajo destinadas a la selección de los escenarios participantes, actividades a desarrollar y la ejecución de eventos de capacitación. Siguiendo la metodología descriptiva, se identificaron los horizontes del perfil del suelo en los 17 escenarios cafetaleros, mediante la construcción de calicatas de 1,20 metro cúbico. De cada horizonte se obtuvo muestras de suelo y se registró en una ficha de campo sus principales características físicas como: profundidad de los horizontes, color y consistencia.

Utilizando en el método de Bouyucos modificado se analizaron las 17 muestras de los escenarios cafetaleros, para el efecto se utilizó una probeta graduada de $250 \mathrm{ml}$, en la cual se colocó $150 \mathrm{~g}$. de suelo seco y triturado, después se agregó agua destilada hasta completar la capacidad de la probeta, luego se agitó por 1 minuto y se dejó reposar por aproximadamente 24 horas [3], determinado el contenido porcentual de las partículas de suelo (arcilla, limo y arena). Una vez obtenido los porcentajes de cada partícula se utilizó el triángulo textural, que es el método de clasificación textural del Departamento de Agricultura de los EEUU (USDA).

Mediante la técnica de observación se realizó un recorrido de la unidad de producción agrícola para identificar el lugar en riesgo de erosión, además de seleccionar el tipo de prácticas de manejo y conservación de suelo a aplicar. 
Construcción de las terrazas individuales. Es una técnica de conservación de suelo ancestral, que permite adecuar racionalmente las laderas de los terrenos con pendientes pronunciadas para el desarrollo de las plantas, y consiste en elaborar terraplenes en la base de plantas formando especies de escalones donde se acumulan la materia orgánica [4].

Elaboración de compost tipo bocashi modificado.- El compost es un bioabono resultante de la descomposición controlada del estiércol y otros materiales orgánicos de la finca, en condiciones adecuadas de humedad y temperatura (Duicela et al. 2003).

Los materiales necesarios para elaborar compost son: Estiércoles de animales: bovinos, porcinos, aves u otros (30\%); Residuos vegetales secos: cáscaras de cacao, maní o fréjol, panca de maíz, tamo de arroz o de fréjol (15$20 \%$ ) Residuos vegetales frescos: basura orgánica doméstica, pseudotallos de plátano, follaje de leguminosas, desperdicios de las cosechas de hortalizas o frutales (50\%); Mulch o tierra de bosque (1-10\%); 300 gramos de levadura y Ceniza o cal (1\%). Para el activado microbiológico, se pone en maceración 1,5 kilos de maíz durante 7 días, en 10 litros de agua. Al séptimo día, moler el maíz, sin botar el agua; añadir 2,0 litros de melaza o 1,0 kilo de panela, agregar 300 gramos de levadura y Mezclar los ingredientes.

Procedimiento. - Colocar los materiales por capas, sobre el nivel del suelo, mezclar los con el uso de una pala, hasta homogenizar el montón, luego humedecer el montículo hasta cerca del punto de saturación. Adicionar al montículo, de manera uniforme, el "activador microbiológico" del compostaje, con el añadido de la levadura. Mezclar nuevamente los materiales del montículo y controlar la uniformidad de la humedad, después se Extiende la masa de tal forma que la altura del montón tenga alrededor de unos 50 centímetros, por la longitud y ancho necesarios. Añadir sobre el montículo, uniformemente, una porción de cal agrícola o ceniza vegetal, para corregir la acidez.

Controlar que el montículo este siempre húmedo; por lo tanto, debe regarse periódicamente. Además, se debe voltear periódicamente el montículo: la primera semana una vez al día; en la segunda semana pasando un día; y, posteriormente, cada siete días, hasta cumplir el proceso. En aproximadamente 12 semanas no se distinguirán los materiales orgánicos procesados, ni se constatará una alta temperatura dentro del montículo. El uso de la levadura reduce el tiempo de compostaje [5].

\section{RESUltados}

Perfiles de suelo. - La visualización y análisis de los perfiles de suelo permitió observar el desarrollo y disposición de los horizontes $\mathrm{O}, \mathrm{A}, \mathrm{B}, \mathrm{B} 1$ y C en la mayoría de los perfiles de suelos. El $53 \%$ de los suelos no presentan horizonte "O" como consecuencia de la erosión debido a la topografía irregular presente en los escenarios cafetaleros. El relieve influye sobre el transporte por gravedad. Los relieves abruptos favorecen la erosión. Además, la diversificación de horizontes en un perfil determina la antigüedad de los suelos (Jordán, 2006). En la tabla 1, se muestran los horizontes presentes en las calicatas construidas en los escenarios cafetaleros.

TABLA I. Profundidad de los horizontes en los perfiles de suelo de los escenarios cafetalero vinculados al proyecto.

\begin{tabular}{|c|c|c|c|c|c|c|c|c|c|c|c|c|c|c|c|c|c|c|c|}
\hline \multirow{2}{*}{$\begin{array}{l}\text { Horizont } \\
\text { Pro- } \\
\text { fundi- } \\
\text { dad en } \\
\text { cm }\end{array}$} & \multicolumn{17}{|c|}{ Número de calicatas } & \multirow[t]{2}{*}{$\mathbf{n}$} & \multirow[t]{2}{*}{$\%$} \\
\hline & 1 & 2 & 3 & 4 & 5 & 6 & 7 & 8 & 9 & 10 & 11 & 12 & 13 & 14 & 15 & 16 & 17 & & \\
\hline $\mathbf{O}$ & 9 & 4 & 2 & & & 3 & & 9 & & 3 & & & & 5 & 13 & 5 & & 9 & $\mathbf{0 , 5 3}$ \\
\hline $\mathbf{A}$ & 20 & 32 & 13 & 12 & 25 & 27 & 64 & 21 & 8 & 24 & 30 & 25 & 15 & 7 & 23 & 25 & 60 & 17 & 1,00 \\
\hline B & 30 & 59 & 20 & 18 & 21 & 28 & 40 & 22 & 47 & 62 & 10 & 50 & & 10 & 41 & 50 & 30 & 16 & 0,94 \\
\hline B1 & 40 & 23 & 47 & 76 & & 13 & & 60 & & & 75 & & & & 43 & & 30 & 9 & $\mathbf{0 , 5 3}$ \\
\hline $\mathrm{C}$ & 20 & & 35 & 10 & 69 & 43 & 10 & & 56 & 21 & & 35 & 90 & 80 & & 25 & & 12 & 0,71 \\
\hline
\end{tabular}

Fuente: Richard Palma y Jesús Pinargote

Se analizó además la profundidad de cada horizonte que conforman los diferentes perfiles, determinándose que el $41,2 \%$ de los suelos resultaron ser profundos $(>100 \mathrm{~cm}$ de profundidad); un 35,3\% suelos moderadamente profundos ( $>60<100 \mathrm{~cm}$ de profundidad) y $23,5 \%$ suelos poco y muy poco profundos (Figura I). 


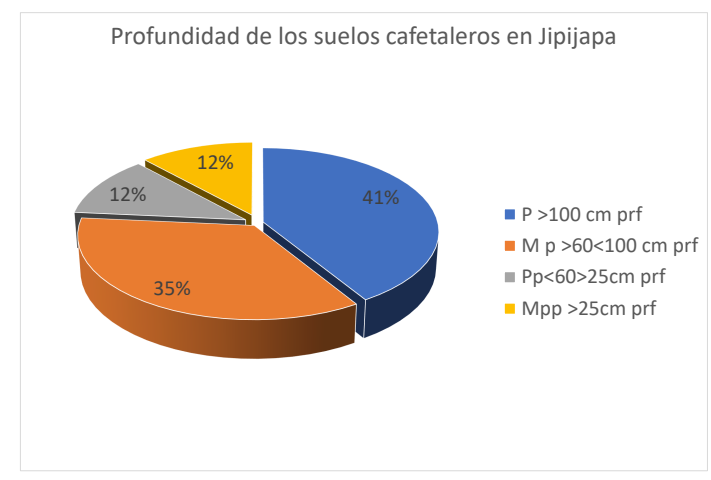

(a)

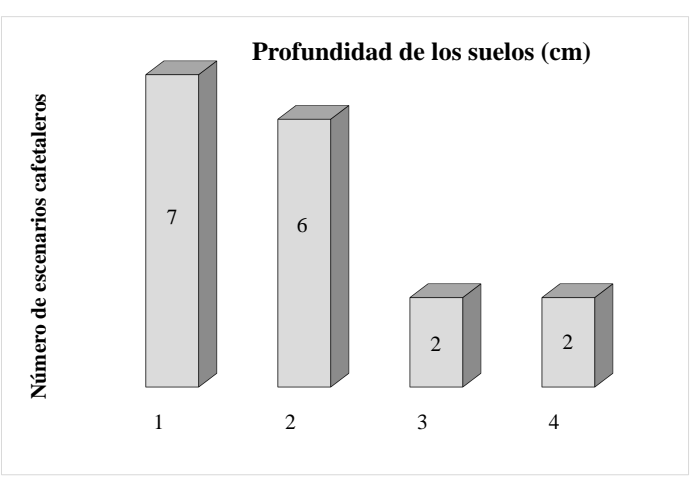

(b)

Fig. 1. Profundidad de los suelos cafetaleros en centímetros

1)Suelos profundos $(\mathrm{P})>100 \mathrm{~cm}$ de profundidad; 2) Suelos moderadamente profundos $(\mathrm{Mp})>60<100 \mathrm{~cm}$ de profundidad; 3 ) suelos poco profundos $(\mathrm{Pp})<60>25 \mathrm{~cm}$ profundidad; 4) Suelos muy poco profundos (Mpp) $<25 \mathrm{~cm}$ de profundidad.

El análisis de textura de los suelos en el horizonte "A", determina que el $76,5 \%$ tienen tendencia franca, predominando ligeramente los francos arcillosos, con el $29,4 \%$, mientras que en el horizonte "B" se establece que el $64,7 \%$ corresponde también a suelos de tendencia franco, predominando al igual que en el horizonte $\mathrm{A}$, los suelos franco arcilloso con el $64,7 \%$, considerados aptos para la agricultura.

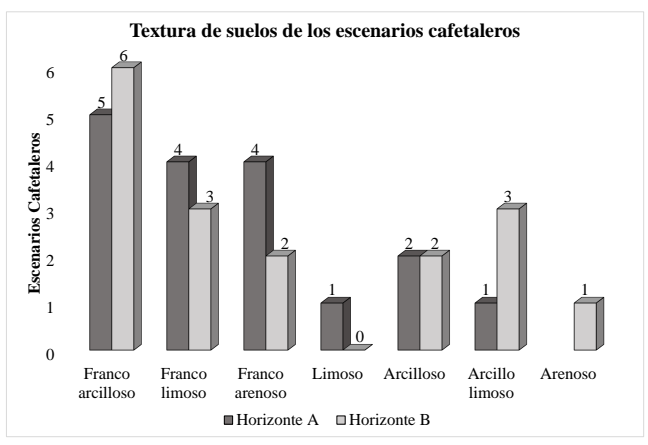

Fig. 2. Textura de los horizontes A y B presente en los perfiles de suelos en los escenarios cafetaleros
Aplicación de prácticas de manejo y conservación de suelo. - Como alternativa de protección contra la erosión de los suelos, en terrenos con pendientes, se construyeron terrazas individuales en cada uno de las 17 fincas vinculadas al proyecto. Además, como estrategia de manejo y recuperación de los suelos degradados en zonas de riesgo se realizó la elaboración de abonos orgánicos, líquidos y sólido, para lo cual se realizó un taller práctico la capacitación pertinente para dichos eventos.

Capacitación. - Se realizaron talleres de capacitación en dos niveles, para estudiantes y para agricultores. En los talleres dirigidos a los agricultores, los estudiantes fueron los facilitadores y contaron con el apoyo y respaldo de los docentes.

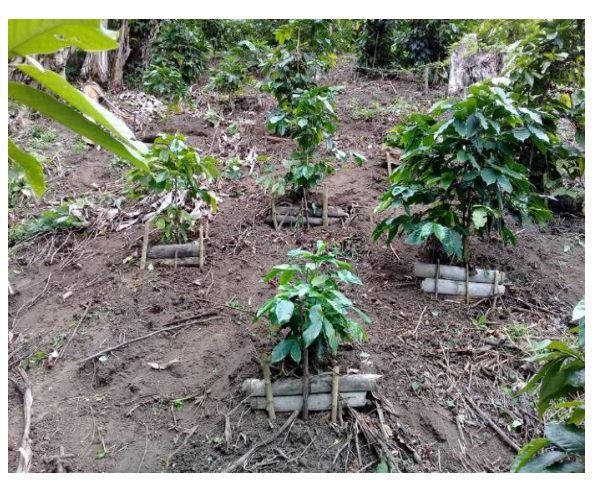

(a)

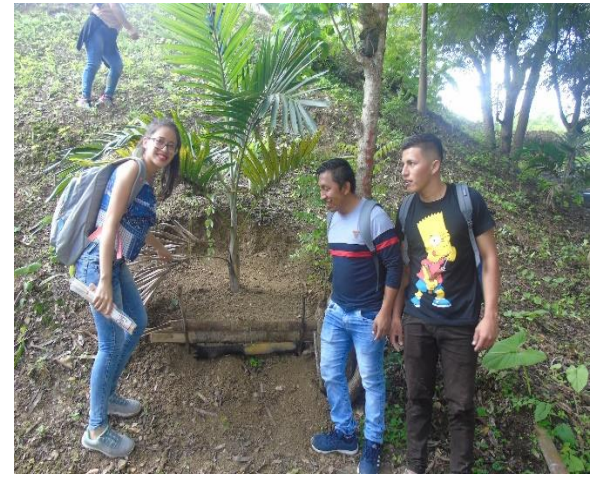

(b)

Fig. 3. Construcción de terrazas individuales 


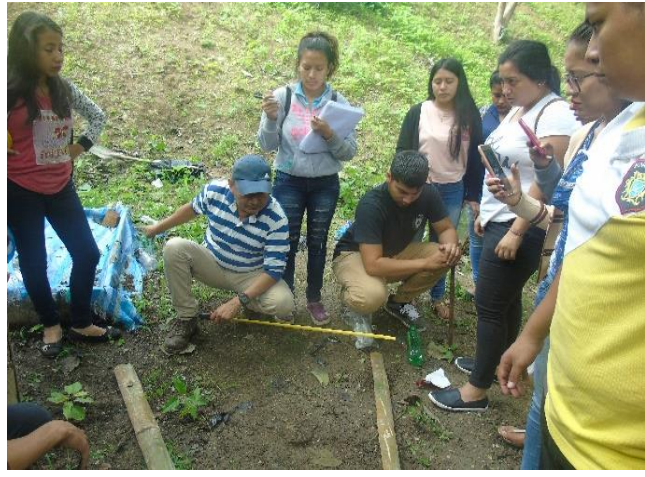

Fig. 4. Capacitación en prácticas de conservación de suelo (trazado con nivel en A)

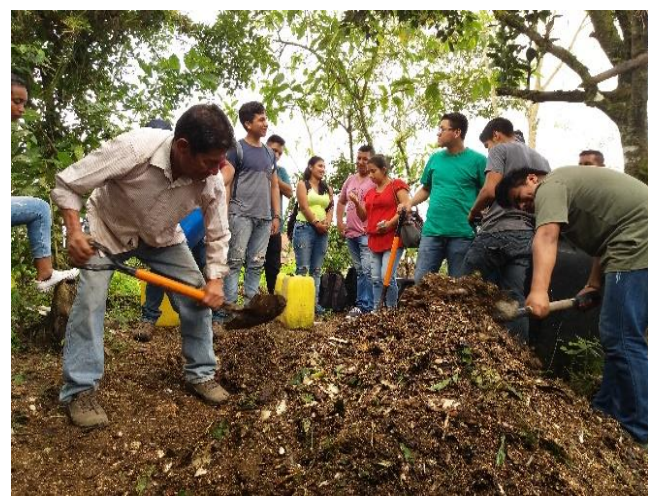

Fig. 5. Capacitación en elaboración de abonos orgánicos (Compost tipo bocashi)

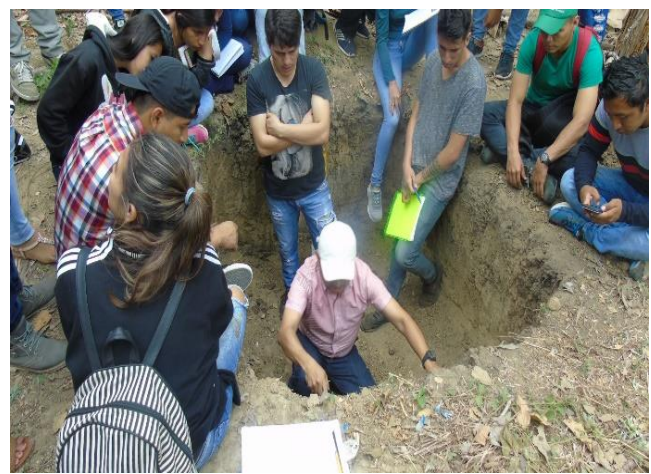

Fig. 6. Capacitación en construcción de calicatas y análisis de perfiles de suelo

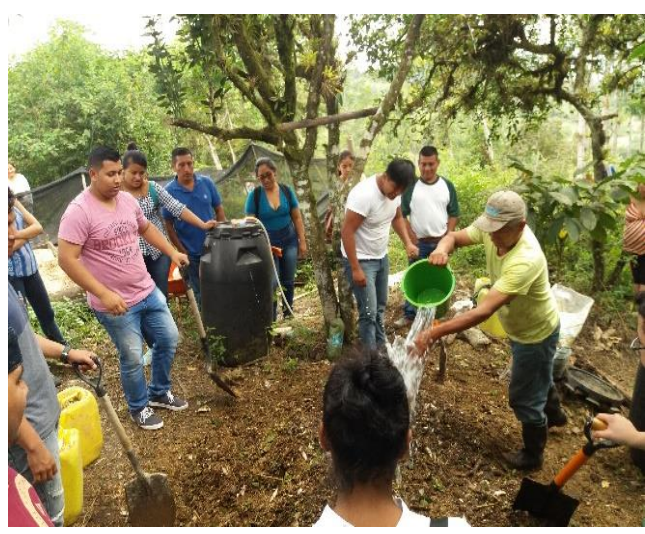

Fig. 7. Capacitación en elaboración de abonos orgánicos (Compost y biol)

\section{CONCLUSIONES}

Mediante calicatas se identificó los perfiles de suelos de las fincas, visualizándose los horizontes presentes. La ausencia del horizonte "O" en varios de los perfiles demuestra la erosión que sufren permanentemente estos suelos.

Los suelos presentan características texturales adecuada para el desarrollo de agricultura, predominando los suelos de tendencia francos.

La participación activa de los agricultores facilita la aplicación de prácticas de manejo y conservación de suelo en las fincas o escenarios cafetaleros vinculados al proyecto.

El trabajo armónico y articulado entre docentes, estudiantes y agricultores fortalecen los ejes sustantivos de la UNESUM, como son: vinculación con la sociedad, investigación y docencia, convierte a la universidad en eje fundamental para el desarrollo de las comunidades.

\section{RECOMENDACIONES}

Se recomienda la implementación de un sistema de trabajo que posibilite transferir esta experiencia a diferentes escenarios de la región, lo que contribuirá a reducir la erosión de los suelos

\section{REFERENCIAS}

[1] Ecured, 2019.

[2] L. Duicela, Manejo Sostenible De Fincas Cafetaleras: Buenas Prácticas en la producción de café arábigo y gestión de la calidad en las organizaciones de productores. Fondo Común para los Productos Básicos (FCPB) y Consejo Cafetalero Nacional (COFENAC). Instituto de Cooperación para la Agricultura (IICAEcuador, segunda edición ed., 2017.

[3] R. Palma, T. Fuentes, L. Ponce, M. Ganchozo, J. Pinargote, and J. Parrales, "Perfil del suelo en la esperanza en la parroquia el anegado del cantón jipijapa," Recimundo. Revista Científica Mundo de la Investigación y el Conocimiento, pp. 1496-1506, enero 2019.

[4] J. Carrasco, F. Squella, J. Riquelme, J. Hirzel, and H. Uribe, Técnicas de Conservación de Suelos, Agua, y vegetación en Territorios Degradados. Región del 
Libertador General Bernardo O'Higgins, CHILE: Instituto de Investigaciones Agropecuarias, 2012.

[5] L. Duicela, R. Corral, C. L, F. Chóez, F. Romero, R. Palma, F. Fernández, A. Macías, D. Farfán, J. Ramírez, L. Zambrano, J. Reyes, and T. Aveiga, Tecnologías para la producción de café orgánico. Manta, EC: COFENAC, PROMSA, 2012.

Recibido: 5 de enero de 2019

Aceptado: 25 de marzo de 2019 